\title{
La construction du raisonnement dans le texte scientifique
}

\author{
Eliane Lousada \\ Université de São Paulo, Brésil \\ elianelousada@uol.com.br \\ Thiago Santos \\ Université de São Paulo, Brésil \\ thiagojorgefs@gmail.com
}

\section{Introduction}

Cet article a pour objectif de présenter une étude préliminaire sur l'apprentissage du genre textuel «projet de recherche » à travers des exemples produits par des étudiants et de futurs chercheurs étant donné qu'ils développent une recherche $\mathrm{d}^{\text {' }}$ Initiation scientifique ${ }^{1}$ ». À travers une analyse textuelle et discursive, nous visons à mieux comprendre l'apprentissage de ce genre essentiel dans la sphère scientifique. Plus précisément, notre article a les objectifs suivants:

a. analyser l'évolution du thème de recherche à travers les parties des projets de recherche;

b. vérifier le mode de construction du raisonnement scientifique, en mobilisant le type de discours théorique (Bronckart, 1999);

c. observer la cohésion textuelle dans les projets de recherche.

Il convient cependant de souligner qu'il s'agit d'une étude en cours et les résultats présentés ne rendent compte que partiellement de nos objectifs généraux de recherche.

Comme le dit Leitão $(2012: 18)$, le genre textuel "projet de recherche » a une grande importance dans le domaine scientifique brésilien, car il est utilisé dans le contexte universitaire pour les processus de sélection comme le doctorat, le master et l'attribution de bourses de recherche aux chercheurs. Il a également acquis une grande importance dans le milieu scolaire, comme un outil pour l'enseignement et l'apprentissage. Cependant, au-delà de ces fonctions, le projet de recherche est généralement le premier genre de la sphère scientifique que l'étudiant d'initiation scientifique doit produire.

En raison de cette particularité, le projet de recherche peut fournir des informations importantes pour la compréhension du développement du raisonnement scientifique. Afin de construire ce raisonnement, les étudiants doivent s'approprier le genre textuel projet de recherche et les aspects linguistiques et discursifs nécessaires dans la mobilisation du discours théorique.

D'après Machado, Lousada et Abreu-Tardelli (2005), les genres textuels scientifiques sont en général enseignés par leur organisation structurelle, sans prendre en compte d'autres aspects qui sont intrinsèques à la constitution de ces genres, comme leurs différentes situations de production et leur organisation linguistique et discursive. Souvent, selon les auteures, ces genres sont exigés des étudiants sans aucun enseignement systématique, soit à l'école soit à l'université. 
Pour atteindre nos objectifs, nous nous référons au modèle théorique l'Interactionnisme socio-discursif ${ }^{2}$ (Bronckart, 1999, 2006, 2008) en ce qui concerne les concepts de texte et de genre textuel, ainsi que la méthode d'analyse textuelle et discursive proposée par ce même cadre théorique. Dans les discussions sur le genre textuel projet de recherche, nous nous appuyons sur les travaux de Machado, Lousada, AbreuTardelli (2005) et Motta-Roth, Hendges (2010). Pour comprendre l'analyse du modèle de l'Interactionnisme socio-discursif, surtout le type de discours théorique, nous utilisons les études de Grize $(1984,1990)$ dans la définition du raisonnement logique qui doit être étudié par l'analyse du contexte de production et de la textualité. Enfin, nous considérons importantes les œuvres de Favero (2012) et Koch (2012) dans notre analyse de la cohésion textuelle dans les projets de recherche.

Pour la compréhension de cette étape notre étude en cours, nous montrerons, d'abord, nos apports théoriques, en détaillant les concepts essentiels sur lesquels nous nous appuyons (notre point 2); puis nous décrirons notre méthodologie, à travers les informations sur les participants de la recherche, le contexte et les données qui sont objets d'analyse (notre point 3). Ensuite, nous passerons à notre analyse partielle des projets de recherche étudiés (notre point 4). Enfin, nous montrerons nos considérations finales.

\section{Cadre Théorique}

Cet article a comme apport théorique principal la théorie de l'Interactionnisme socio-discursif (Bronckart 1999, 2006, 2008) et les études de Machado, Lousada, Abreu-Tardelli (2005) et Motta-Roth, Hendges (2010) sur le genre textuel projet de recherche. Par la suite, nous allons présenter les concepts théoriques de base de l'Interactionnisme socio-discursif (ISD) pour ensuite traiter des recherches sur le genre textuel que nous étudions. Nous compléterons le cadre théorique de l'ISD par des études qui approfondissent quelques questions importantes pour l'analyse du genre textuel "projet de recherche », comme, par exemple, les écrits de Favero (2012) et Koch (2012) sur la cohésion nominale.

D'après Bronckart (2003 : 56), l'ISD comprend que la conscience et les fonctions psychologiques supérieures de l'homme (surtout la pensée et le langage) sont produites par l'appropriation et après par l'intériorisation des propriétés des différentes formations sociales dans lesquelles s'inscrivent tous les êtres humains. Ce processus d'intériorisation se réalise à son tour dans le cadre structurel qui constitue l'action humaine et les productions sémiotiques, en particulier les productions verbales.

Les formations sociales se développent toujours dans un contexte physique, économique et historique spécifique. Dans ces formations sont créés des règles, des normes et des valeurs qui régulent les interactions parmi les membres des groupes, à travers une division de travail toujours conflictuelle (Bronckart, 2006 : 209). Étant donné que les interactions verbales orales ou écrites gèrent et représentent les moyens d'interaction d'une formation sociale donnée, les textes sont aussi régulés par ces moyens.

$\mathrm{Au}$ sein de chaque groupe social, il existe des textes (oraux ou écrits) qui sont les correspondants empiriques d'une action langagière et sont produits avec les ressources d'une langue naturelle. En tant qu'action langagière, les caractéristiques de composition des textes dépendent des propriétés des interactions, de l'activité qui les a générés ainsi que des conditions socio-historiques de leur production (Bronckart, 2008: 113). Dans la mesure où chaque texte appartient à un ensemble de textes, ils appartiennent, selon l'ISD, à un genre textuel. Ainsi, dans cette approche, on considère que les genres textuels sont des instruments symboliques disponibles dans une société, mais seront considérés comme cela quand ils deviennent des instruments psychologiques appropriés et utiles pour agir avec la langue (Machado, Lousada, $2010: 625$ ). 
Les études de l'ISD sur un grand ensemble de textes ont mené au développement d'un modèle d'analyse textuelle selon lequel chaque texte a une organisation hiérarchique à trois niveaux qui interagissent. C'est ce que nous présenterons par la suite.

\subsection{Le premier niveau d'analyse pour I'ISD}

Dans cette partie, nous présenterons les aspects qui doivent être considérés au niveau de la infra-structure textuelle, comme le plan global du contenu thématique - en abordant la manières dont les informations sont disposées dans le texte - et aussi les types de discours, c'est-à-dire, d'une part [en] l'établissement des coordonnées temporelles organisant la mise en scène des états-événements-actions sémiotisés, d'autre part [en] la clarification du type de rapport existant entre les instances actorielles sémiotisées et celles impliquées dans l'action langagière à l'origine d'un texte (BRONCKART, 2008).

\subsubsection{Le plan global du contenu thématique}

A un niveau plus profond, chaque texte a une organisation du contenu et de l'expression de la production du langage. Premièrement, nous observons la planification du contenu thématique, à savoir, les thèmes et les éléments de connaissance mobilisés. Ensuite, nous analysons les types de discours et les séquences textuelles présents dans le texte.

Pour analyser le plan global du contenu thématique du genre textuel projet de recherche, nous avons utilisé les études de Machado, Lousada, Abreu-Tardelli-(2005) et Motta-Roth, Hendges (2010). Comme ces deux études considèrent l'importance du contexte de production dans la textualisation et aussi l'organisation du langage par les genres des textes, elles peuvent servir de base pour notre analyse basée sur l'ISD. Selon les deux études citées, le projet de recherche doit avoir les éléments suivants:

- identification ou données du projet: ici les données les plus importantes sont le titre de la recherche (qui doit exprimer précisément le thème ou problème dont le projet s'occupe); le domaine de la recherche, l'auteur et le directeur de recherche; l'institution.

- Problèmes, hypothèses et questions: présentation de la problématique de recherche ou une question à laquelle le chercheur veut répondre.

- Justification: ici il faut montrer la pertinence et l'originalité du projet, en soulignant son innovation. Cette partie est très argumentative, elle doit convaincre le lecteur de l'importance de la recherche (Motta-Roth Hendges, $2010: 56$ ).

- Objectif général et objectif spécifique: selon Machado, Lousada, Abreu-Tardelli (2005:84), l'objet à traiter doit être précisé au début de l'introduction, doit être accompagné du récit de l'itinéraire suivi pour la délimitation du sujet. L'objectif général est ce que l'on souhaitait obtenir grâce à la recherche. L'objectif spécifique précise le contenu de l'objectif général, exprimé par des éléments distincts ( $a$ et $b$, par exemple).

- Synthèse de la littérature pertinente: un résumé des textes lus, montrant les concepts pertinents centraux pour la recherche. Pour Motta-Roth et Hendges (2010:57), la recherche scientifique est un service communautaire d'une communauté scientifique donnée avec ses objets d'étude, ses raisonnements, ses outils théoriques. 
- Méthodologie: explicitation des procédures adoptées; nature des données; description des procédures de recueil des données collectées.

- Résultats attendus/impacts: cette partie est l'une des plus argumentatives, parce que l'objectif est de convaincre le lecteur que les résultats attendus seront importants et utiles.

- Échéancier: c'est la division temporelle des étapes de la recherche dans la durée du travail dans son ensemble.

- Budget: explicitation des sources financières de la recherche, si c'est le cas.

- Bibliographie à la fin du projet de recherche, toutes les références citées au long du document (en particulier celles du cadre théorique) doivent être indiquées et classées par ordre alphabétique.

\subsubsection{Les types de discours}

Après avoir présenté de manière succincte le plan global du projet de recherche, revenons au niveau plus profond de l'organisation des textes proposée par l'ISD.

Pour comprendre le concept de types de discours, Il faut comprendre les deux opérations fondamentales qui les définissent. Dans un premier temps, Bronckart (1999), à la suite de Weinrich (1973), oppose le monde raconté au monde commenté, c'est-à-dire le monde du RACONTER et le monde de l'EXPOSER, se référant à la construction de la coordination des opérations qui organisent le contenu thématique d'un texte : elles sont disjointes si elles sont explicitement placées à distance de la situation de production du texte (événements passés et futurs), ou conjointe, si cette distance n'est pas effectuée.

Dans la deuxième opération, qui est basée sur les études énonciatives de Benveniste (2006), on observe si le texte est impliqué ou autonome. Si les actions exprimées dans le texte sont liées à la production du texte et à sa situation de production, il y a une relation d'implication, sinon s'établit une relation d'autonomie.

La présence de ces deux sous-ensembles (RACONTER et EXPOSER/ impliqué et autonome) définissent quatre types de discours (Bronckart, 1999):

- Monde du RACONTER impliqué (récit interactif)

- Monde du RACONTER autonome (narration)

- Monde de l'EXPOSER impliqué (discours interactif)

- Monde de l'EXPOSER autonome (discours théorique)

Les types de discours sont attestés dans toutes les langues, mais chacune mobilise certaines ressources linguistiques pour les exprimer. La pratique et l'appropriation de ces quatre types de discours, selon l'ISD (Bronckart 2008: 91), construisent et développent les différentes formes de raisonnement humain, comme le raisonnement causal/temporel (Ricoeur, 1983) dans les mondes du RACONTER (narration et récit interactif); raisonnement du sens commun dans le discours interactif (Roulet, 1985) et le raisonnement logique-argumentatif dans le discours théorique (Grize, 1984). Lorsque l'agent (re)produit un type de discours, il doit procéder à la planification interne des segments concernés, en apprenant ainsi à activer ces processus, mentaux et langagiers, qui sont les raisonnements (Bronckart, 2006). 
En ce qui concerne le discours théorique et le raisonnement logique, Bronckart affirme que ce type de discours a toujours tendance à l'autonomie par rapport aux paramètres de l'action langagière, c'est-à-dire, à mobiliser les opérations cognitives dans un système qui se déroule dans le successif, qui est généralisé, abstrait et dépend de circonstances particulières (Bronckart, Bulea, 2008).

Pour Grize (1984), un raisonnement peut être un texte, une suite d'expressions, une activité, l'opération d'une pensée qui résout un problème ou une structure formée de relations logico-sémantiques. Selon cet auteur, le produit logique doit être observé dans sa construction à la suite de l'utilisation des ressources d'une langue naturelle. De ce point de vue, le raisonnement logique est construit différemment, par exemple, de la logique mathématique dans laquelle, à partir de certaines prémisses données comme véritables, il est possible de dire si un argument est logique ou non.

Dans un texte construit en discours théorique, dans lequel il y a une quête de la conceptualisation dans un ordre logique des opérations cognitives, il faut analyser les opérations textuelles effectuées afin de vérifier le degré de formalisation du raisonnement et dire s'il s'agit d'un raisonnement formel ou informel.

Selon Grize (1990 : 56), le raisonnement formel est considéré comme un domaine fermé, élaboré à partir de principes. Les éléments du domaine sont organisés entre eux, et cette combinaison est similaire à un "calcul". En revanche, le raisonnement non formel contient des conclusions et donc n'a pas de linéarisation dans un domaine fermé, une séparation des affirmations ordonnées. Par conséquent, le raisonnement non formel fonctionne avec des notions et non sur les concepts, raison pour la laquelle il est moins exigeant, plus fluide, plus imprécis que le raisonnement formel (Grize, 1990 :59 - 62).

Dans la relation entre ces deux types de raisonnement, Grize (1990:62) comprend que le passage du type formel au non formel sert à transformer les idées en concepts, allant du plus ou moins précis à l'exactement déterminé, de la pensée commune à la pensée scientifique.

\subsection{Les mécanismes de textualisation}

Dans le deuxième niveau de l'analyse textuelle de l'ISD, on vérifie les mécanismes de textualisation, qui confèrent la cohérence thématique au texte par diverses relations établies entre les unités et structures mobilisées afin de faire progresser le texte. A ce niveau, trois mécanismes sont observés: les connexions, la cohésion nominale et verbale. La cohésion textuelle a la fonction d'introduire de nouveaux éléments d'information et d'assurer leur redressement dans le texte (Bronckart, 1999).

En combinant la cohésion nominale avec les types de discours, Bronckart (1999:271) affirme que les relations de cohésion du discours théorique sont plus fréquentes parce qu'il y a une articulation des arguments plus abstraits, donc ils sont habituellement associés par les relations de coréférence. Cependant, dans un texte cette relation peut être effacée, avec l'absence de cohésion.

Afin d'approfondir l'analyse de la cohésion textuelle, importante dans notre étude, nous avons eu recours à d'autres chercheurs qui se sont penchés sur cette question, tels que Favero (2012) et Koch (2012), car ils proposent des catégories qui permettent de mieux analyser les textes. En ce qui concerne les fonctions de la cohésion du texte, Favero (2012) propose un reclassement des études existantes. Ainsi, sont proposés trois types de cohésion: référentielle, de récurrence et séquentielle. Ces études sont importantes pour notre travail, car elles mettent en relief la corrélation entre les activités humaines et les productions langagières, en partant de la théorie de l'activité de Vygotski et Leontiev (KOCH, 2012 : 11-12). Et pour cette raison, elles peuvent être rapprochées de l'ISD.

À propos de la cohésion référentielle, selon Favero (2012: 18), il y a certains éléments de langue qui ont pour fonction d'établir une référence à quelque chose de nécessaire à leur interprétation. La référence est 
un premier degré d'abstraction dans lequel le lecteur rapporte un signe déterminé à un objet tel qu'il est perçu dans la culture dans laquelle il vit.

La cohésion de récurrence a pour fonction de faire marcher, progresser la circulation de l'information à travers la reprise des structures, des objets et des séquences qui mènent le discours en avant. Par récurrence, d'anciennes informations sont liées à des nouvelles. Voici des cas de cohésion par récurrence: la récurrence de termes, parallélisme, paraphrase, segments phonologiques et caractéristiques prosodiques.

La récurrence des termes a la fonction d'intensification et est un moyen de créer ou d'assurer le flux du texte. En traitant de ce cas de cohésion, Koch (2012:55) comprend que, bien qu'il y ait répétition de termes, il ne peut y avoir identité de sens entre eux, parce que chacun apporte de nouvelles instructions de signification concernant l'antérieure.

Le dernier type de cohésion étudié par Favero (2012) est la séquentielle. L'effet de l'utilisation de ce type est également de faire progresser le texte dans le niveau informationnel, cependant pas par récurrence mais à travers les éléments temporels et les éléments qui établissent des connexions (connecteurs), la causalité, l'explication et d'autres.

Le dernier niveau d'analyse, selon Bronckart (1999: 319), concerne les mécanismes énonciatifs. Ils offrent une grande contribution à l'établissement de la cohérence interactive, pragmatique ou configurationnelle du texte et guident également le destinataire en interpréter le contenu thématique. D'après Lousada (2010), ces mécanismes donnent au texte une clarification dialogique à travers la voix et modalisations.

Les trois niveaux textuels décrits ci-dessus sont déterminées par le contexte de production, dans lequel émerge chaque action langagière. Dans le domaine scientifique, certains genres textuels doivent être appropriés par les chercheurs qui développent des travaux de recherche. Ainsi, la structure textuelle de ces genres est dépendante des éléments extra-linguistiques, qui sont déterminés par les activités sociales auxquelles ils sont articulés, et aussi par les situations de communication dans lesquelles les genres sont nécessaires (Bronckart, 2007).

Pour la compréhension du contexte extra-linguistique de production, il faut d'abord comprendre l'activité sociale (Bronckart, 1999 : 30) dans laquelle le texte est produit et où il circule parce que, comme indique Bronckart (1999), les activités sociales organisent les comportements langagiers ou non et constituent le principe explicatif des actions attribuées à une personne.

\section{Méthodologie}

Dans cette partie, nous allons présenter les procédures méthodologiques qui ont été utilisées pour le développement de notre étude. Dans ce but, nous allons d'abord indiquer les participants impliqués dans ce travail et le contexte de notre étude, pour éclairer l'ensemble des données que nous avons, et enfin, nous allons montrer nos procédures d'analyse.

\subsection{Le contexte et les participants}

Dans cette étude, nous avons comme participants des étudiants de la Licence en Lettres de la Faculté de Philosophie, Lettres et Sciences Humaines de l'Université de São Paulo (FFLCH-USP) ${ }^{3}$. Ces étudiants font leurs études dans la licence en langue française et langue portugaise et ont écrit leurs projets de recherche à différents niveaux du cours de langue française, mais la plupart d'entre eux à la deuxième ou troisième année d'étude. Les projets de recherche ont été développés au sein de la chaire d'Etudes 
Linguistiques, Littéraires et de Traduction en français, du Département de Lettres Modernes de l'USP, où les thèmes de recherche sont: les relations France- Brésil; les problèmes de critique de la littérature française; la traduction et la traduction de la littérature; la didactique et l'apprentissage du français ; l'analyse des textes et des discours. Parmi ces possibilités, nous avons choisi des projets qui appartiennent aux lignes de recherche suivantes: "Didactique du français comme langue étrangère" et "Études des langues, des cultures et des textes".

Les étudiants qui ont participé à notre étude ont écrit un projet de recherche pour la première fois dans le cadre de l'initiation scientifique, et n'avaient pas de connaissances préalables sur ce genre. Étant donné qu'il y a très peu d'orientations fournies par la faculté sur le genre textuel projet de recherche, les étudiants avaient de grandes difficultés au moment de la préparation et de l'écriture.

\subsection{Méthode d'analyse du texte}

Comme nous l'avons mentionné, nous avons analysé neuf projets de recherche produits par des étudiants de Lettres qui développent une recherche au niveau de l'Initiation scientifique. Les projets de recherche PR1, PR3, PR4, PR6 et PR8 avaient comme cadre théorique l'Interactionnisme socio-discursif; mais les projets PR2, PR5, PR7 et PR9 ont été écrits à partir de différents cadres conceptuels. Ces projets ont été écrits en différentes années, comme le montre le tableau ci-dessous:

\begin{tabular}{|c|c|c|c|}
\hline $\mathbf{2 0 1 0}$ & $\mathbf{2 0 1 1}$ & $\mathbf{2 0 1 2}$ & $\mathbf{2 0 1 3}$ \\
\hline PR1 et PR2 & PR4 et PR5 & PR6 et PR7 & PR8 et PR9 \\
PR3 (fragment 5) & & & \\
\hline
\end{tabular}

Tableau 1: années où les projets de recherches ont été écrits.

\subsection{Méthodes d'analyse des données}

Dans un premier temps, nous avons analysé le contexte socio-subjectif où les projets de recherche ont été élaborés pour observer ensuite le plan global des contenus thématiques (Machado, Lousada, AbreuTardelli, 2005; Motta-Roth, Hendges, 2010) et les types de discours, en mettant l'accent sur la construction du raisonnement formel et informel par la textualisation (Grize, 1984, 1990). Ainsi, nous avons étudié les projets de recherche dans leur macrostructure textuelle.

Après, nous avons analysé les mécanismes de cohésion nominale et de textualisation, afin de montrer comment le projet de recherche est construit dans la récupération et la circulation de l'information contenue dans ces textes. Pour ce faire, nous avons observé surtout la cohésion de récurrence (Fávero, 2012; Koch 2012).

\section{L'analyse des projets de recherche}

Nous exposerons d'emblée l'étude du contexte socio-interactionnel dans lequel les neuf projets de recherche ont été produits pour présenter par la suite l'analyse du plan global du contenu thématique, de l'usage du discours théorique pour la construction du raisonnement logique et aussi des aspects de cohésion textuelle présents dans les textes.

\subsection{Analyse du contexte socio-subjectif de production}


Les projets de recherche ont été produits dans un lieu social qui est la Faculté de Philosophie, Lettres et Sciences Humaines de l'USP, qui possède un programme d'Initiation à recherche scientifique (IC) géré par la Commission de recherche de la Faculté de Lettres (CPQ/FFLCH) qui établit les dates d'enregistrement des projets, gère la présentation des rapports de recherche, et distribue les ressources financières, c'est-à-dire la gestion bureaucratique de l'IC. Le projet de recherche, est ainsi une condition préalable pour l'enregistrement officiel de l'étudiant dans le programme d'Initiation à recherche scientifique et est indispensable pour la demande d'une bourse de recherche.

À travers l'appel d'offre de l'IC, la CPQ fournit des lignes directrices pour la rédaction du projet de recherche, qui doit contenir: Introduction, Objectif, Méthodologie et Références. Les projets doivent avoir un maximum de quatre pages (avec la page de garde). Pour la rédaction du projet de recherche, les étudiants n'ont pas d'autres consignes sauf celles données dans l'appel d'offre. Bien que la taille du projet ne soit pas grande, les étudiants ont des difficultés parce qu'ils n'ont pas de connaissance de ce genre.

Les projets que nous avons examinés ont été rédigés par des étudiants de la licence en Lettres de la FFLCH/USP, en particulier les étudiants de la licence en langue française et portugaise, qui ont commencé la recherche dans l'Initiation Scientifique dans les premières années du cours de lettres. Ces étudiants sont intéressés principalement aux questions liées à l'enseignement du français comme langue étrangère (FLE) et à l'analyse des textes et des discours. Aucun de ces étudiants n'avait écrit un projet de recherche auparavant, ils n'avaient donc aucun contact avec cette pratique textuelle.

Le premier destinataire des projets analysés était le professeur directeur de recherche, qui a lu et dirigé la réécriture du texte. A partir des orientations et suggestions du superviseur, les étudiants ont changé les projets, qui étaient encadrés par les consignes de la $\mathrm{CPQ} / \mathrm{FFLCH}$. Après trois ou quatre versions, plus ou moins, les neufs projets ont été envoyés à la $\mathrm{CPQ}$, alors il existe plusieurs versions de ces textes.

\subsection{Analyse du plan global du contenu thématique}

Comme nous avons déjà expliqué, les projets que nous avons analysés devaient être formatés selon les lignes directrices de l'appel à l'initiation scientifique. Comme il s'agit du premier projet rédigé par les étudiants, les textes ont montré des variations en ce qui concerne le format prévu.

Plus de la moitié n'ont pas de titres et deux de ces projets n'ont pas de page de garde : nous avons un projet dont le titre n'a pas été explicité et un autre qui commence par l'introduction.

Comme nous allons montrer dans les analyses, les parties du projet de recherche ${ }^{4}$ sont organisées selon les premières idées que les étudiants ont à propos de ce genre textuel et sur le "travail scientifique". Justement parce que les étudiants ne maitrisent pas toutes les étapes de la recherche, les titres ne sont pas inclus. Dans ce premier moment de l'écriture et de l'apprentissage du projet de recherche, les étudiants n'ont pas encore développé un raisonnement formel de tout processus nécessaire pour le déroulement de la recherche scientifique, ce qui est un facteur de complication pour choisir un titre qui résume le travail.

Ce que nous venons d'affirmer peut être compris si nous prenons comme exemple l'un des titres des projets analysés dans notre corpus. Le titre de ce projet est "Séquence didactique et FLE", qui est très large et ne permet pas d'avoir un aperçu de la particularité de cette recherche. Les mots du titre pourraient être utilisés pour remplir les "mots-clés " ou utilisés dans le titre lui-même, mais disposés de manière à souligner l'objectif de la recherche.

En analysant les Introductions des projets nous avons aussi trouvé des particularités qui découlent du fait qu'il s'agit de l'apprentissage de ce genre textuel par les étudiants. Pour présenter les objectifs du projet, 
nous avons identifié deux tendances: soit il y a un objectif général à travailler, soit il y a un objectif encore incohérent ou incomplet sur l'étude envisagée. A titre d'exemple:

(1) Este projeto de iniciação científica pretende trabalhar com a aquisição do francês como língua estrangeira, abordando o uso do estudo de um gênero textual escrito para o trabalho na zonade desenvolvimento proximal [...] Assim a partir da análise dos dados coletados poderemos sugerir novos posicionamentos para melhorar o ensino do francês como língua estrangeira. (PR2)

Ce projet d'initiation à la recherche a l'intention de travailler avec l'acquisition du français comme langue étrangère, en abordant l'usage de l'étude d'un genre textuel écrit pour le travail dans la zone de développement proximale [...] Ainsi, à partir de l'analyse des données recueillies, nous pouvons suggérer de nouveaux positionnements pour améliorer l'enseignement du français comme langue étrangère. (PR2)

Comme on le voit dans l'extrait ci-dessus, l'objectif de la recherche est d'améliorer l'enseignement du français comme langue étrangère (FLE) dans son ensemble. Cet objectif est très grand et il ne serait pas possible dans une recherche d'initiation scientifique car cela demanderait des données qui montrent les lacunes de l'enseignement du FLE, ainsi que des connaissances des recherches qui ont traité ce sujet. Toutefois, nous comprenons que l'objectif fixé dans le projet en question est le résultat de la représentation que l'étudiant se fait de son expérience en tant qu'étudiant de la licence en Lettres (option langue française), c'est-à-dire, étant lui-même un étudiant de français comme langue étrangère.

Pour traiter la deuxième tendance que nous avons trouvée dans l'écriture des objectifs de recherche, nous allons montrer deux morceaux qui nous aideront dans notre réflexion:

(2) Com este projeto, visamos a estudar o desenvolvimento da expressão escrita dos alunos, através de produções escritas organizadas a partir de gêneros textuais e que têm como eixo condutor as atividades linguageiras expor, relatar e descrever ações. (PR6)

Avec ce projet, nous visons à étudier le développement de l'expression écrite des étudiants, à travers des productions écrites organisées à partir des genres textuels et qui ont comme fil conducteur les activités langagières exposer, relater, décrire des actions. (PR6).

(3) Essa pesquisa consiste em escolher gêneros textuais apropriados para o ensino da língua francesa para os alunos do $1^{\circ}$ ano de aprendizagem de tal idioma, que tenham o mínimo ou nenhum conhecimento prévio do francês. (PR5)

Cette recherche consiste à choisir des genres textuels appropriés pour l'enseignement de la langue française pour les étudiants de la première année d'apprentissage de telle langue, qui aient peu [de] ou aucune connaissance préalable du français. (PR8).

Dans le fragment (2), on note que l'objectif visé est l'étude des genres textuels de l'ordre de l'EXPOSER et du RELATER dans des textes produits par des étudiants universitaires dans le deuxième semestre en langue française. Cependant, les genres textuels qui seront produits par les étudiants et qui seront analysés ne sont pas explicités. De même, dans le fragment (3), l'intention est étudier les genres appropriés pour l'enseignement du FLE dans un contexte universitaire, toutefois les genres qui seront travaillés ne sont pas explicités. 
Pour justifier la pertinence du travail, nous estimons que, contrairement à ce que l'on entend par justification dans une conception canonique, les projets que nous avons examinés ont fait remarquer que l'objectif de la recherche sélectionnée vient du contexte de production décrit ci-dessus. Étant donné que les étudiants sont à la licence en lettres langue française les projets sont justifiés en raison de leurs propres expériences dans ce contexte, comme nous pouvons le voir dans les extraits ci-dessous:

(4) O presente projeto tem por objetivo o estudo da produção escrita de alunos universitários implicados no aprendizado do francês como língua estrangeira, tendo em vista o lugar privilegiado da escrita como instrumento de desenvolvimento das capacidades de linguagem e, portanto, da comunicação em si mesma. (PR8)

Le présent projet a pour objectif l'étude de la production écrite des étudiants universitaires impliqués dans l'enseignement du français comme langue étrangère, en prenant en compte le lieu privilégié de l'écriture comme instrument de développement des capacités langagières, et donc de la communication elle-même. (PR8)

(5) A escolha pelo gênero editorial foi motivada pela dificuldade existente, em alguns estudantes, em lidar com a "seqüência argumentativa" (Bronckart, 1999), contudo, o conhecimento pelo aluno do modo como se organiza o gênero editorial lhe permitirá tanto apropriar-se de tal gênero, como valer-se dele como instrumento. (PR3)

Le choix pour le genre éditorial a été motivé par la difficulté existante, du côté de quelques étudiants, à comprendre la "séquence argumentative" (Bronckart, 1999), néanmoins, la connaissance par l'étudiant de la façon dont s'organise le genre éditorial lui permettra l'appropriation de tel genre et aussi son utilisation en tant qu'instrument. (PR3)

Dans les extraits ci-dessus, dans lesquels les étudiants justifient la pertinence de leurs projets, on note que c'est l'implication dans le contexte de l'apprentissage du FLE qui fait ressortir un possible objet de recherche, soit l'enseignement et l'apprentissage de la langue française dans la forme écrite ou orale, soit la relation entre la langue et la culture ou les difficultés rencontrées par les étudiants eux-mêmes. C'est donc le fait de proposer une étude qui permet d'étudier leur propre contexte d'apprentissage du FLE qui justifie la pertinence du projet. Ce sont les difficultés des étudiants que nous voyons dans le fragment (5) qui motivent le projet de recherche. Comme il y a une difficulté dans l'apprentissage de l'argumentation en français, une étude sur cette question est nécessaire pour résoudre cette complication notée dans l'apprentissage.

Dans les deux justifications, nous ne voyons pas de connaissances par les étudiants des recherches antérieures sur les sujets abordés, parce que précisément parce qu'ils ne connaissent pas encore ces études le projet ne se justifie que par leurs expériences en tant que étudiants en Lettres, intéressés par les questions d'enseignement et l'apprentissage du FLE.

Dans les projets analysés, la méthodologie décrite est également liée au contexte dans lequel les étudiants se trouvent. Pour comprendre comment cette relation fonctionne, nous allons utiliser les extraits suivants:

(6) Para responder à pergunta " $A$ " dos objetivos, será coletado um corpus produzido por alunos do primeiro ano da habilitação em francês do curso de Letras da Universidade de São Paulo. (PR8) 
Pour répondre à la question " $\mathrm{A}$ " des objectifs, un corpus produit par les étudiants de la première année de la licence en Lettres (langue française) de l’Université de São Paulo. (PR8)

(7) Para responder à primeira questão, coletaremos vários textos pertencentes aos gêneros que serão estudados pelos alunos do segundo semestre do bacharelado de Francês/Português do DLM-USP. (PR6)

Pour répondre à la première question, nous recueillerons plusieurs textes appartenant aux genres qui sont étudiés par les étudiants du deuxième semestre de la licence de Français/Portugais do DLM-USP. (PR6)

Si l'on analyse le contexte de la collecte de données, on observe que ces lieux sont très proches des étudiants, et ils sont souvent impliqués dans ce contexte, car l'objet de l'analyse sont les textes produits par d'autres étudiants qui sont dans les semestres précédents du cours.

Les projets montrent une utilisation considérable des sources secondaires pour synthétiser les textes et concepts théoriques pertinents pour la recherche, comme nous pouvons constater dans l'extrait suivant:

(8) Tal proposta de pesquisa foi fundamentada na noção de gênero feita por Bakhtin e retomada por pesquisadores comoBronckart (1997) e Dolz \& Schneuwly (1996):

"a cada tipo de atividade humana que implica o uso da linguagem corresponde enunciados particulares, os gêneros do discurso. Enquanto que os gêneros são relativamente estáveis, os textos que os materializam são extremamente variáveis e maleáveis."

(Bronckart, 1997; Dolz \& Schneuwly, 1996 apud Lousada, 2002)

Desse modo, somos levados a concluir que "como cada situação de uso da íngua se realiza verbalmente através de um gênero, a capacidade de comunicação depende da maior ou menor domínio que se tem do gênero em questão" (Dolz \& Schnewly, 1996 apud Lousada, 2002).

Também foi adotada a noção de gênero como ferramenta que atua no processo de aprendizagem (Schneuwly \& Dolz, 2004 apud ___ ), onde o gênero é encarado como verdadeiro instrumento para o desenvolvimento dos três tipos de capacidade da linguagem (capacidade de ação, discursiva e lingüístico-discursiva) as quais são os fundamentos para o domínio de uma língua. (Dolz \& Schneuwly, 1996 apud Abreu-Tardelli, 2004). (PR4)

Telle proposition de recherche a été fondée sur la notion de genre faite par Bakhtine e reprise par des chercheurs comme Bronckart (1997) et Dolz \& Schneuwly (1996):

"a cada tipo de atividade humana que implica o uso da linguagem corresponde enunciados particulares, os gêneros do discurso. Enquanto que os gêneros são relativamente estáveis, os textos que os materializam são extremamente variáveis e maleáveis."

(Bronckart, 1997; Dolz \& Schneuwly, 1996 apud Lousada, 2002)

De cette façon, nous sommes amenés à conclure que "étant donné que chaque situation de l'usage de la langue se réalise verbalement à travers le genre, la capacité de communication dépend de la maîtrise appropriée du genre en question" (Dolz \& Schnewly, 1996 apud Lousada, 2002). 


\begin{abstract}
De plus, nous avons adopté la notion de genre comme outil qui contribue au processus d'apprentissage (Schneuwly \& Dolz, 2004 apud ), où le genre est vu comme un vrai instrument pour le développement des trois types de capacités langagières (action, discursive et linguistique) lesquelles sont les fondements pour la maitrise d'une langue (Dolz \& Schneuwly, 1996 apud Abreu-Tardelli, 2004). (PR4)
\end{abstract}

Nous voyons l'utilisation constante de la forme apud pour montrer une citation secondaire. Cela est le résultat de la non connaissance des sources primaires du domaine de recherche investigué. En fait, l'utilisation de sources secondaires est d'une grande importance pour la compréhension des sources primaires, car elles permettent que ces sources soient comprises par l'application dans les contextes de recherche. Dans les projets analysés, cependant, la remarquable utilisation de ces sources est une tentative d'organisation des idées, en prenant en compte les premières lectures faites.

\title{
4.3 Analyse des types de discours: le raisonnement logique formel et non formel
}

Dans l'analyse de l'utilisation du discours théorique, nous observons un processus de formalisation du raisonnement logique, en soulignant à travers ces analyses linguistiques comment il est textualisé. Nous avons trouvé la textualisation du raisonnement non formel à différents moments des projets de recherche, dans la synthèse de la littérature pertinente et aussi dans la méthodologie. Les fragments ci-dessous le montrent.

(9) Para o ensino da língua portuguesa, os PCN definem que a importância do uso da competência discursiva, é fazer o sujeito saber utilizar a língua de forma variada, produzindo diferentes efeitos de sentido e adequar o texto a diferentes situações de interlocução oral e escrita, ou seja, saber utilizar os diferentes tipos de gêneros em diferentes situações. Dessa forma, podemos inferir que a relação histórico-social do indivíduo com a linguagem ajuda-o a delimitar e ao mesmo tempo ampliar sua vivência com o mundo. A partir da fala, as crianças podem inferir e questionar os conceitos e premissas estabelecidos como padrões sociais. Nessa perspectiva, a aplicabilidade do gênero como fator de mediação torna-se essencial. (PR1)

Pour l'enseignement de la langue portugaise, les $\mathrm{PCN}^{5}$ définissent que l'importance de l'usage de la compétence discursive est de faire développer chez les sujets la capacité d' utiliser la langue de formes variées, en produisant différents effets de sens et en adaptant les textes aux différentes situations d'interlocution orale et écrite, c'est-à-dire, savoir utiliser les différents types de genres dans différentes situations. De cette façon, nous pouvons inférer que les relations historiquessociales des individus avec le langage peuvent contribuer à définir et simultanément à élargir leur expérience avec le monde. À partir de la parole, les enfants peuvent inférer et remettre en question les concepts et les hypothèses établis comme des normes sociales. Dans cette perspective, l'applicabilité du genre textuel comme facteur de médiation devient essentielle. (PR1)

(10) Ao escolher o gênero textual como um instrumento que contribui para o desenvolvimento das capacidades de linguagem, adotamos também o conceito de sequência didática, proposto por Schneuwly e Dolz (2004) e que tem o objetivo de trabalhar um gênero textual com os alunos. Segundo esses autores, a sequência didática que a professora executará entre a produção inicial e a produção final desse gênero. (PR2) 
Dans le choix du genre textuel comme un instrument qui contribue au développement des capacités du langage, nous adaptons aussi le concept de séquence didactique, proposée par Schneuwly e Dolz (2004) et qui a le but de travailler un genre textuel avec les élèves. Selon ces auteurs, la séquence didactique que le professeur fera entre la production initiale et la production finale de ce genre. (PR2)

Dans l'extrait (9), l'étudiant, d'abord, définie le concept de compétence discursif, en expliquant que son développement fait que les élèves comprennent et sachent utiliser la langue en divers contextes, de manières diverses, soit dans la modalité orale, soit écrite, à travers des genres textuels. Après cela, on observe le connecteur "De cette façon" ("Dessa forma" en portugais) qui établit une relation de causalité entre les deux phrases, mais un lien qui fait émerger un raisonnement non formel du caractère historique et social de la théorie vygotskienne adoptée par l'étudiant dans sa recherche. Par la phrase introduite par "De cette façon" on ne cherche pas la relation entre la compétence discursive préconisée dans les PCNs et la conception du langage chez Vygotski.

A son tour, dans le fragment (10), le raisonnement logique non formel est introduit par la cohésion établie par le connecteur "Selon ces auteurs" ("Segundo esses autores" en portugais) qui montre l'appropriation du concept de séquence didactique par l'étudiant au moment où il considère que la séquence se restreint seulement aux activités mises en marche entre les deux productions textuelles. Pour que les apprenants développent leurs capacités langagières, il faut considérer la production initiale comme une étape essentielle dans le processus de la séquence didactique, car c'est le moment où l'enseignant vérifie la zone de développement réelle des élèves pour ensuite créer des ateliers pour remplir les lacunes d'apprentissage et aussi la zone de développement potentiel.

(11) Também foi adotada a noção de gênero como ferramenta que atua no processo de aprendizagem (Schneuwly \& Dolz, 2004 apud ), onde o gênero é encarado como verdadeiro instrumento para o desenvolvimento dos três tipos de capacidade da linguagem (ação, discursiva e lingüístico) as quais são os fundamentos para o domínio de uma língua. (Dolz \& Schneuwly, 1996 apud Abreu-Tardelli, 2004). (PR4)

De plus, nous avons adopté la notion de genre comme outil qui contribue au processus d'apprentissage (Schneuwly \& Dolz, 2004 apud ), où le genre est vu comme un vrai instrument pour le développement de trois types de capacités langagières (action, discursive et linguistique) lesquelles sont les fondements pour la maîtrise d'une langue (Dolz \& Schneuwly, 1996 apud Abreu-Tardelli, 2004). (PR4)

(12) Uma das fontes teóricas desse trabalho são as ideias de Vygotski sobre interação social (Apud Davis e Oliveira, 1994), na qual o indivíduo se constitui em uma sociedade e em um contexto histórico, os quais exercem suas influências sobre o mesmo, contribuindo em uma relação dialética entre o indivíduo e o meio, para construir e produzir conhecimento.

Outro conceito importante da teoria vygostkiana que será utilizado neste trabalho é o de mediação, segundo o qual a relação entre o homem e o meio se faz por meio de ferramentas materiais ou simbólicas (Oliveira, 2003).

$[\ldots]$ 


\begin{abstract}
Outra referência teórica deste trabalho são os conceitos da Perspective actionnelle, abordagem proposta pelo Quadro Europeu Comum de Referência para as línguas (CECR. Conseil de l'Europe, 2001), para a qual os "usuários e os aprendizes de uma língua são tidos como atores sociais". (PR5)
\end{abstract}

L'une des sources théoriques de ce travail sont les idées de Vygotski sur l'interaction sociale (Apud Davis e Oliveira, 1994), dans laquelle l'individu se constitue dans une société et dans un contexte historique, qui exercent leurs influences sur lui, en contribuant à une relation dialectique entre l'individu et le milieu, afin de construire et produire la connaissance.

Un autre concept important de la théorie vygotskienne qui sera utilisé dans ce travail est celui de médiation, selon laquelle la relation entre l'homme et le milieu se fait par les outils matériaux ou symboliques (Oliveira, 2003).

$[\ldots]$

Une autre référence théorique de ce travail sont les concepts de la Perspective actionnelle, démarche proposée par le Cadre Européen Commun de Référence pour les langues (CECR, Conseil de l'Europe, 2001), pour lequel les "usagers et apprentis d'une langue sont des acteurs sociaux". (PR5)

Dans l'extrait (11), le connecteur qui nous montre le raisonnement logique non formel est le «De plus» ( «Também» en portugais). Il introduit un paragraphe qui est la suite du paragraphe antérieur où on avait la définition du concept de genre textuel, à partir de Bakhtine, en expliquant sa relative stabilité et sa matérialisation dans les textes. Pourtant, dans le paragraphe commencé par «Aussi» une autre notion est ajoutée à la recherche, celle de genre textuel en tant qu'outil pour l'enseignement de langues, par lequel trois capacités langagières peuvent se développer (l'étudiant fait une erreur, car les capacités sont «d'action, discursive, linguistique-discursive - pas «linguistique » seulement), mais, au moment d' expliquer le concept de genre textuel d'après Bakhtine, l'étudiant n'établit pas de rapport logique entre ce concept et la notion d'« outil », qui pourrait être mieux élucidée par la théorie vygotskyenne sur le concept de langage et apprentissage.

Dans le fragment (12), dans la synthèse des hypothèses théoriques d'un autre projet, nous pouvons identifier l'utilisation du raisonnement non formel par l'absence d'éléments de cohésion. Pour comprendre ce problème, nous mettons en évidence trois segments, qui montrent le choix de l'étudiant de travailler avec deux concepts de la théorie de Vygotski et des concepts de la perspective actionnelle (Conseil de l'Europe, 2001). Dans un premier temps, l'étudiant explique l'utilisation du concept d'interaction comme une relation dialectique entre l'individu et l'environnement, pour construire et pour produire des connaissances. L'étudiant affirme que c'est précisément à cet égard que l'homme développe des outils pour ses actions, c'est-à-dire, il s'agit d'une relation médiatisée. Le concept de médiation est également utilisé dans les travaux du PR5, mais il n'y a pas de lien explicite entre les deux concepts, celui d'《interaction» et celui de «médiation». Nous identifions donc un raisonnement non formel aussi. L'utilisation répétée de l'élément lexical «autre»pour introduire les concepts utilisés, qui gère la cohésion de récurrence de ce terme, ne permet pas d'établir la relation de sens entre eux, comme constaté dans les études de Koch (2012).

Lorsque nous faisons attention au dernier segment souligné, nous notons également qu'il n'existe pas de relation cohérente entre les deux concepts de Vygotski (2009) sélectionnés avec les autres concepts de la perspective actionnelle, issus d'un autre cadre théorique. 
(13) Para ambos, todo gênero é constituído de diversos elementos que representam os objetos de ensino gradativo que devem ser aplicados pelo professor a fim de que o aprendiz desenvolva suas capacidades de linguagem e, mais que apreender uma modalidade de texto, possa expandir os novos savoir-faire para diversas realizações comunicativas que partilhem elementos constitutivos em comum. Assim, no processo de aprendizagem, faz-se necessário compreender quais conteúdos são dizíveis em determinado gênero, assim como entender qual é sua estrutura comunicativa particular, formada por um conjunto de unidades linguísticas, de sequências textuais e pelos tipos de discurso que constroem o texto.

Pour les deux, tout genre est constitué par divers éléments qui représentent les objets d'enseignement continu qui doivent être appliqués par le professeur afin que les étudiants développent leurs capacités langagières et, plus qu'apprendre une modalité de texte, puissent élargir les nouveaux savoir-faire pour diverses réalisations communicatives qui partagent des éléments constitutifs en commun. Ainsi, dans le processus d'apprentissage, il faut comprendre quels contenus peuvent être dits dans un genre déterminé, de même que comprendre quelle est sa structure communicative particulière, formée par un ensemble d'unités linguistiques de séquences textuelles et par les types de discours qui construisent le texte.

(14) O trabalho que aqui se propõe buscará justamente compreender o modo como leitores específicos - os franceses - têm acesso ao trabalho do poeta brasileiro, e como se deu a expansão de sua obra literária no país que o influenciou e inspirou. Para isso, deve-se realizar o levantamento dos poemas traduzidos para a língua francesa, baseando-se em textos teóricos sobre a recepção da tradução e em textos propagados pela crítica literária, além de se estudar o contexto em que foram traduzidos os poemas em questão. (PR7)

Le travail proposé ici cherche à comprendre précisément la façon dont les lecteurs spécifiques les français -ont accès à l'œuvre du poète brésilien [Carlos Drummond de Andrade], et la manière d'expansion de son œuvre littéraire dans le pays qui a lui influencé et inspiré. Pour cela, il faut faire un relevé des poètes traduits vers le français, en s'appuyant sur des textes théoriques sur la réception des textes en traduction et sur les textes propagés par la critique littéraire, outre étudier le contexte dans lequel ont été traduits les poèmes en question. (PR7)

Le fragment (13) appartient à la partie de synthèse de la bibliographie théorique de la recherche, dans laquelle l'étudiant voulait étudier l'enseignement du français langue étrangères par les genres textuels de l'ordre de l'exposer et du relater. Pour cela, il a abordé la question des genres discursifs et textuels chez Bakhtine et Bronckart, en soulignant l'importance de la situation de communication dans l'analyse textuelle. Toutefois, dans cet extrait, au moment d'aborder l'enseignement et l'apprentissage par les genres textuels, il a considéré seulement le développement des capacités langagières concernant l'analyse strictement textuelle et non le perfectionnement de la capacité d'action, selon Schneuwly \& Dolz (2004), qui concerne l'utilisation d'un genre textuel dans un contexte donné. Le connecteur "Ainsi » ( « Assim », en portugais) le montre. On observe donc la présence d'un raisonnement non formel, car la méthode d'analyse du contexte de production par la capacité d'action a été considérée par l'étudiant en tant que présupposé théorique non lié à la pratique d'enseignement/apprentissage par les genres textuels.

Dans l'extrait (14) l'étudiant fait des changements dans son projet de recherche, après avoir eu des commentaires de sa directrice de recherche à propos de la manière dont il présentait l'objectif du travail. Selon elle, il devrait améliorer cela. Ainsi, en considérant le commentaire, l'étudiant, à travers le connecteur «Pour cela » («Para isso », en portugais), a explicité un peu plus sur son objectif et il a 
abordé aussi, bien que de manière générale, le concept de «réception de la traduction », qui est central dans sa recherche. Nous remarquons ainsi un raisonnement logique encore non formel sur ce concept, étant donné que l'étudiant faisait ces premières lectures du cadre théorique.

(15) A atual pesquisa fixa-se na vertente teórica do Interacionismo Sociodiscursivo (Bronckart, 2003), que visa a mostrar o papel fundador da linguagem e sobretudo do funcionamento discurso/da atividade discursiva no desenvolvimento humano. Apoiando-se no conceito de Zona de Desenvolvimento Proximal, um estágio pelo qual o indivíduo ainda não tem autonomia para encontrar soluções sozinho, precisando da contribuição de outro [...] (PR8)

Cette recherche se base sur la théorie de l'Interactionnisme socio-discursif (Bronckart, 2005), qui vise à montrer le rôle fondateur du langage et surtout du fonctionnement discursif/de l'activité discursive dans le développement humain. En s'appuyant sur le concept de Zone de Développement Proximal, un stage dans lequel l'individu n'a pas encore d'autonomie pour trouver des solutions lui-même, ayant besoin d'une contribution de l'autre [...]. (PR8)

(16) Como bem determina o linguista francês, para produzir sentido, a informação implica um processo de "produção de discurso em situação de comunicação" (CHARRADEAU, 2010, pp. 33-34). Como sabemos, a informação é pura enunciação. Pois bem, este é o tema central deste trabalho, que se propõe a, por meio de Análise do Discurso, descrever o percurso linguístico pelo qual jornais franceses e brasileiros passaram para construir uma personagem "a partir do zero", por assim dizer. (PR9)

Comme bien le détermine le linguiste français, pour produire sens, l'information implique un processus de "production de discours en situation de communication". Comme nous le savons, l'information est pure énonciation. Voici, ceci est le thème central de ce travail, qui se propose à travers l'Analyse du Discours, de décrire le parcours linguistique par lequel les journaux français et brésiliens ont passé pour construire un personnage "partir du zéro", pour ainsi dire. (PR9)

Dans le fragment (15) du PR8, qui n'avait pas de titre, l'étudiant présente deux cadres théoriques importants pour sa recherche: la théorie de l'Interactionnisme socio-discursif et le concept de zone de développement proximal de Vygotski. La partie que nous avons soulignée dans l'extrait (15) du projet montre clairement ces deux choix théoriques. Cependant, il n'y aucun élément de cohésion qui établit une relation entre les deux choix : la ZPD selon Vygotski et l'ISD. Ceci aurait été possible si l'étudiant savait que l'ISD a comme une des sources principales les études sur le développement de Vygotski. Ainsi, pour expliquer les concepts théoriques qui seront utilisés, l'étudiant développe un raisonnement non formel, car il y a un implicite entre les deux concepts, qui probablement ne serait pas compris par tous les lecteurs de ce projet.

Dans l'extrait (16) l'étudiant met en évidence le fait que l'information dans la théorie sémiolinguistique implique un processus discursif dans une situation d'énonciation. Après cette clarification, l'étudiant a écrit "Comme nous le savons, l'information est pure énonciation" ("Como sabemos, a informação é pura enunciação"), qui n'a aucune relation logique avec la période précédente, à cause de l'absence d'un connecteur entre les affirmations. En plus, ce raisonnement logique non formel nous fait observer l'appropriation de l'étudiant du concept d' "information" dans la théorie qu'il a utilisé dans sa recherche.

\section{Considérations finales}


Guidé par nos objectifs, nous avons montré les particularités du genre textuel "projet de recherche » rédigé par les étudiants brésiliens de recherche scientifique. Ces projet ont été analysés par rapport à l'élaboration du plan global du contenu thématique et à la formalisation du raisonnement logique dans le discours théorique. Nous avons également souligné certaines questions relatives à la cohésion textuelle, en particulier les difficultés à maitriser la cohésion de récurrence.

Comme nous l'avons montré, le premier contact avec l'activité scientifique a souvent lieu à travers la rédaction d'un projet de recherche. Ainsi, ce genre contient les représentations initiales des étudiants au sujet de cette situation de production et par conséquent révèle leurs premières représentations construites dans ces textes à propos du métier de " chercheur".

En ce sens, notre travail a visé à analyser les neuf projets de recherche au niveau macro-structurel, en observant certaines fonctionnalités de ces textes afin de comprendre l'apprentissage à la fois du genre projet de recherche et de la recherche elle-même, en observant un point spécifique d'un raisonnement logique dans le discours théorique, à travers la cohésion textuelle. Nous espérons ainsi avoir contribué à la recherche existante sur le genre textuel «projet de recherche » et surtout aux études qui se concentrent sur le rôle de la textualité dans la construction du raisonnement logique et, par conséquent, du discours scientifique.

Notre article montre l'importance de l'enseignement des genres textuels scientifiques dans le milieu universitaire, en visant au développement et à l'appropriation du discours théorique et, ainsi, du raisonnement logique et argumentatif. Dans ce sens, l'apprentissage épistémique (Vygotski, 2009; Bota, 2011) devient nécessaire pour comprendre comment se passe l'apprentissage des genres textuels scientifiques et des concepts, par exemple, à travers la cohésion textuelle qui, souvent, est considérée comme déjà apprise par les étudiants de l'initiation à recherche. Il convient de souligner que dans le contexte de la Faculté de Lettres de l'USP il n'y a pas de disciplines qui abordent cette question, d'où l'intérêt de notre travail.

Il s'agit néanmoins de résultats partiels d'une recherche en cours. A ce stade, nous n'avons pu qu'amorcer des éléments d'analyse qui montrent un cheminement possible et, nous le croyons, fructueux pour la recherche. Il reste cependant plusieurs questions à prendre en compte, dont la plus importante est probablement la constitution du corpus qui contient à présent cinq projets (sur neuf) basés sur la même théorie que nous utilisons pour l'analyse (l'ISD). Une des prochaines étapes de notre recherche sera donc l'élargissement du corpus ou la vérification des résultats sur des projets de recherche proposés à partir d'autres cadres théoriques, afin d'assurer la crédibilité de nos propos.

\section{Références bibliographiques}

Benveniste, E. (2006). Problemas de Linguística Geral I. Campinas: Pontes.

Bota, C. (2011). Savoirs textes et apprentissages en milieu universitaire: Pour une analyse socio-discursive de travaux de validation pour les cours. Thèse de doctorat. Université de Genève, Genève.

Bronckart, J-P. (1999). Atividade de linguagem, textos e discursos: por um Interacionismo Sociodiscursivo. São Paulo: Educ.

Bronckart, J-P. (2003). Gêneros textuais, tipos de discursos, e operações psicolinguísticas.Revista de Estudos da Linguagem, v.11, 49-69.

Bronckart, J-P. (2006). Atividade de linguagem, discurso e desenvolvimento humano. Campinas, Mercado de Letras. 
Bronckart, J-P. (2007). "L'activité langagière, la langue et le signe, comme organisateurs du développement humain". Langage et société, v.3, 57-68.

Bronckart, J-P. (2008).O agir nos discursos: das concepções teóricas às concepções dos trabalhadores. Campinas : Mercado de letras.

Bronckart, J-P. (2008). "Genres de textes, types de discours et « degrés » de langue. Hommage à François Rastier". Texto!, v. XIII, nº 1 .

Bronckart, J-P ; bulea, E. (2008). As potencialidades praxiológicas e epistêmicas dos (tipos de) discursos. SCRIPTA, v. 12 , p. $42-83$

Conseil de l'europe. (2001). Cadre Européen Commun de Référence pour les Langues. Paris: Didier.

Fávero, L. L. (2012). Coesão e Coerência Textuais. São Paulo: Ática.

Grize, J-B. (1984).Sémiologie du raisonnement. Berne: Peter Lang.

Grize, J-B. (1990). Logique et langage. Paris: Ophrys.

Koch, I. G. V. (2012). A Coesão Textual. São Paulo: Contexto.

Leitão, P. D. V. (2012). A apreensão do projeto de pesquisa por alunos do curso de Licenciatura em Letras. Dissertação de Mestrado (Mestrado em Estudos Linguísticos) Universidade Federal de Pernambuco, João Pessoa.

LOUSADA, E. G. (2010). A abordagem do Interacionismo Sociodiscursivo para a análise de textos. Abordagens metodológicas em estudos discursivos. São Paulo: Editora Paulistana.

Machado, A. R.; Lousada, E. G.; Abreu-Tardelli, L. S. (2005). Planejar gêneros acadêmicos. São Paulo: Parábola.

Machado, A. R.; Lousada, E. G. (2010). A apropriação de gêneros textuais pelo professor: em direção ao desenvolvimento pessoal e à evolução do "métier". Ling. (dis)curso, n.3, 619-633.

Motta-Roth, D., Hendges, G. R. (2010). Produção textual na universidade. São Paulo: Parábola Editorial.

Ricoeur, P. (1983).Temps et récit I. Paris: Seuil.

Roulet, E. (1985). L'articulation du discours en français contemporain. Berne: Peter Lang.

Vygotski, L. S. (2009). A Construção do pensamento e da linguagem. São Paulo: Martins Fontes.

Weinrich, H. (1973). Le temps. Paris: Seuil.

\footnotetext{
${ }^{1} \mathrm{Au}$ Brésil, les étudiants universitaires peuvent proposer des projets de recherche qui sont subventionnés par une bourse du gouvernement, pendant une année. Le but du programme du gouvernement est qu'ils apprennent à développer une recherche. Ce programme gouvernemental s'intitule «Initiation Scientifique ».

${ }^{2}$ Dorénavant ISD.

${ }^{3}$ La licence en Lettres à l'Université de São Paulo dure 5 ans.

${ }^{4}$ Projet de recherche (PR1, PR2...)/

${ }^{5} \mathrm{PCN}$ : Paramètres curriculaires nationaux.
} 